\title{
Finger Actions Sensing-Based Robot Motion Authoring System
}

\author{
Kwang-Ho Seok and Yoon Sang Kim \\ Department of Computer Science and Engineering, \\ Korea University of Technology and Education, \\ 330-708 Cheonan, Chungnam, South Korea \\ yoonsang@koreatech.ac.kr
}

\begin{abstract}
This paper proposes a finger actions sensing-based robot motion authoring system. Based on finger actions sensing and their recognition, the proposed robot motion authoring system allows users easily to create and control robot motion acording tothe number and events of fingers. Furthermore, the system can be used to simutate user-created robot contents in the $3 D$ virtual environment. This allows the users to not only viex the adthoring process in real time but also transmit the final authored content The effectiveness of the proposed motion authoring system was verified based on various motion authoring simulations of an industrial robot.
\end{abstract}

Keywords: finger actions sensing, cobot motion authoring

\section{Introduction}

As the increasing number of applications adopts intelligence, and thus robot requires a new type of robot software environment that allows user to author various commands for robots. Robots must become easier to use for general users in order to expand the scope of applications of intelligent robots in our everyday life. Based on this request, a number of studies are being conducted regarding new types of user-friendly and intuitive robot motion authoring that can render various motions [1-2]. Most of user-made robot motions are based on authoring (the robothotion created and edited by user) using motion capture data [3-4]. There have been studies on how to define a framework for automatically finding primitives for human body gestures [5]. Also, there are research projects actively taking place in humanrobot interaction, thanks to the progress that has been made in vision technology [6-8]. In particular, esearch on gesture recognition using kinect and camera sensor based remote human-robot interaction system is actively being carried out [9-10]. And there are other studies proposing algorithms to improve hand gesture recognition [11-12].

However, user-intuitive or user-friendly robot command authoring system that focus on facilitating general users are still rare. The lack of user-intuitive or user-friendly tools is likely to create a barrier for providing robot services that correspond with the preferences and demands of general users including children, the elderly and housewives, who are expected to be the major clients in the service robot industry.

This paper proposes a finger actions sensing-based robot motion authoring system. Based on finger actions sensing and their recognition, the proposed robot motion authoring system allows users easily to create and control robot motion according to the number and events of fingers. Furthermore, the system can be used to simulate user-created robot contents in the 3D virtual environment. This allows the users to not only view the authoring process in real time but also transmit the final authored contents. The effectiveness of the proposed motion 
authoring system was verified based on various motion authoring simulations of an industrial robot.

\section{Proposed Robot Motion Authoring System}

This paper proposes a robot motion authoring based on finger actions sensing. The proposed robot motion authoring system consisted of a PC camera, a robot motion authoring tool, and an actual robot. Figure 1 illustrates the overall process of the robot motion authoring implemented for this study.

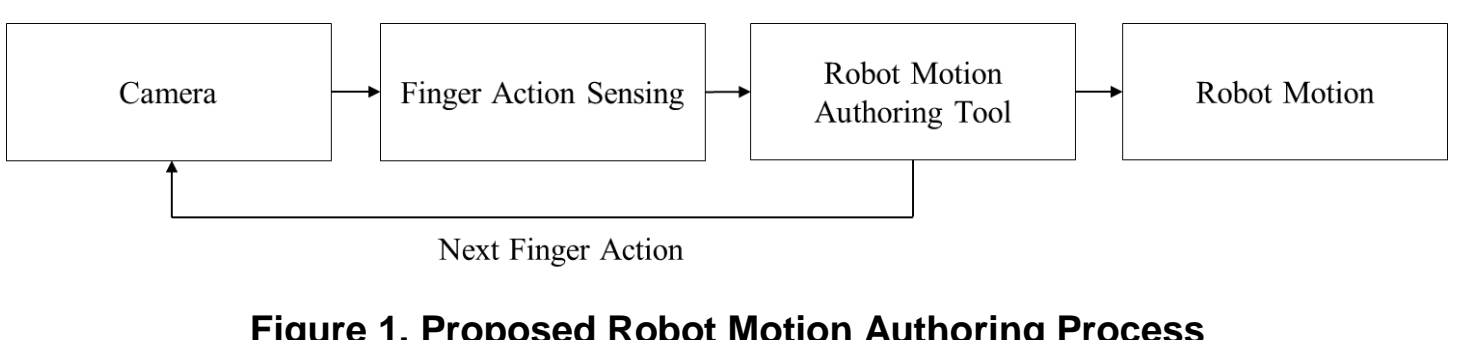

There are a number of techniques for finger áctions sensing, which is being used in various fields. With the exception of language, the hand is most frequently used for human communication among our body parts such 18 hands, eyes, mouth, arms and legs. Figure 2 show various finger actions?

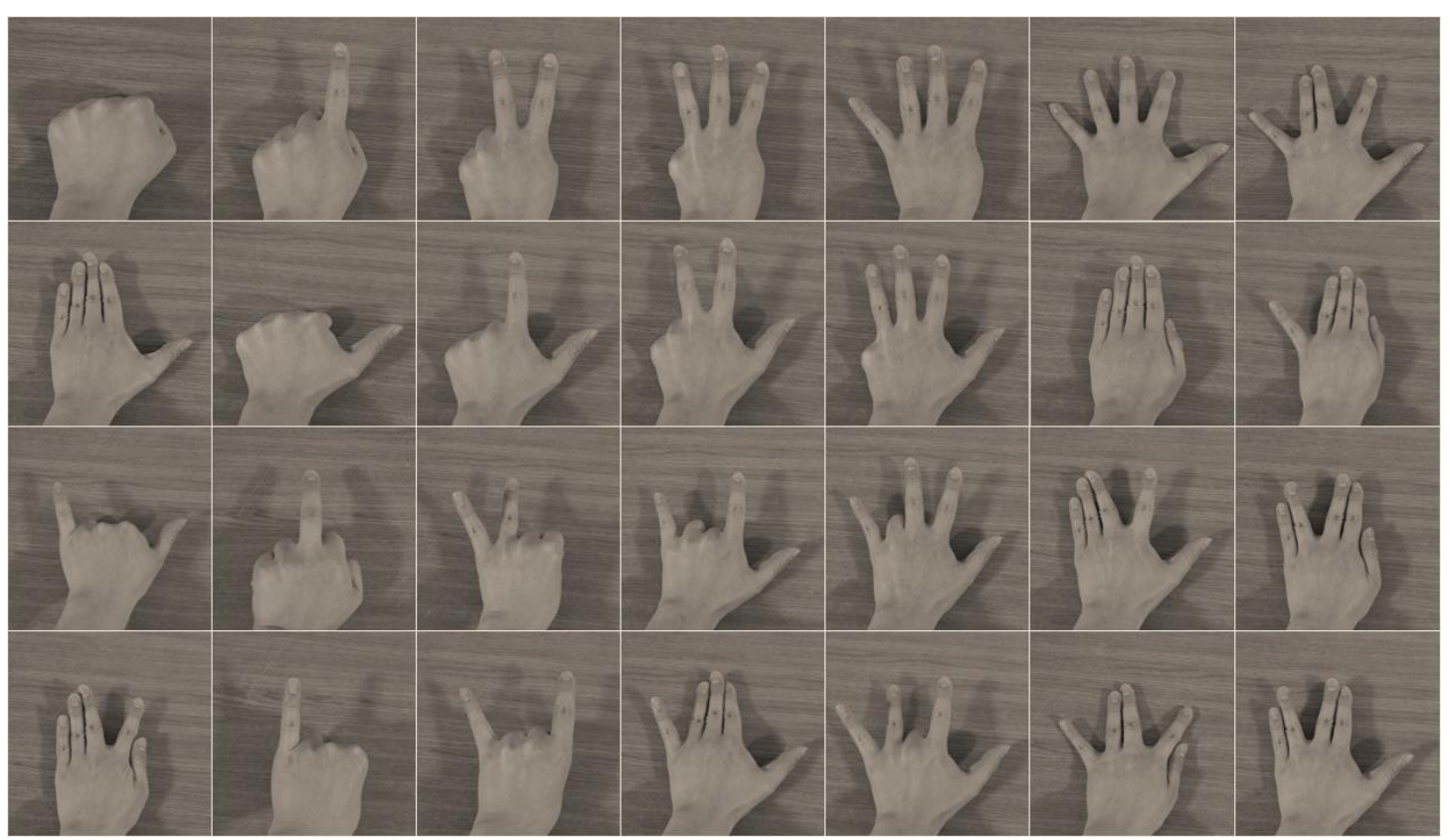

Figure 2. Various Finger Actions

In this study, finger recognition unit is implemented using color values scheme. The finger recognition unit first converts RGB colors into gray scales and $\mathrm{YCrCb}$ for binary representation. Then the region inside the hand is filled by masking and noise is removed. The binary image is examined in 25-pixel units. If sum of 1's examined is greater than ten, every digit is set to 1 . With the image obtained by masking performed 
by the finger recognition unit, the center of the hand can be calculated to identify hand's location as well as the hand region furthest from the center. In this paper, a motion authoring tool capable of creating and controlling robot motions is implemented using the finger actions sensing. The motion authoring tool consists of five panels: the finger actions sensing panel, mode panel, view panel, communication panel and data panel (Figure 3).

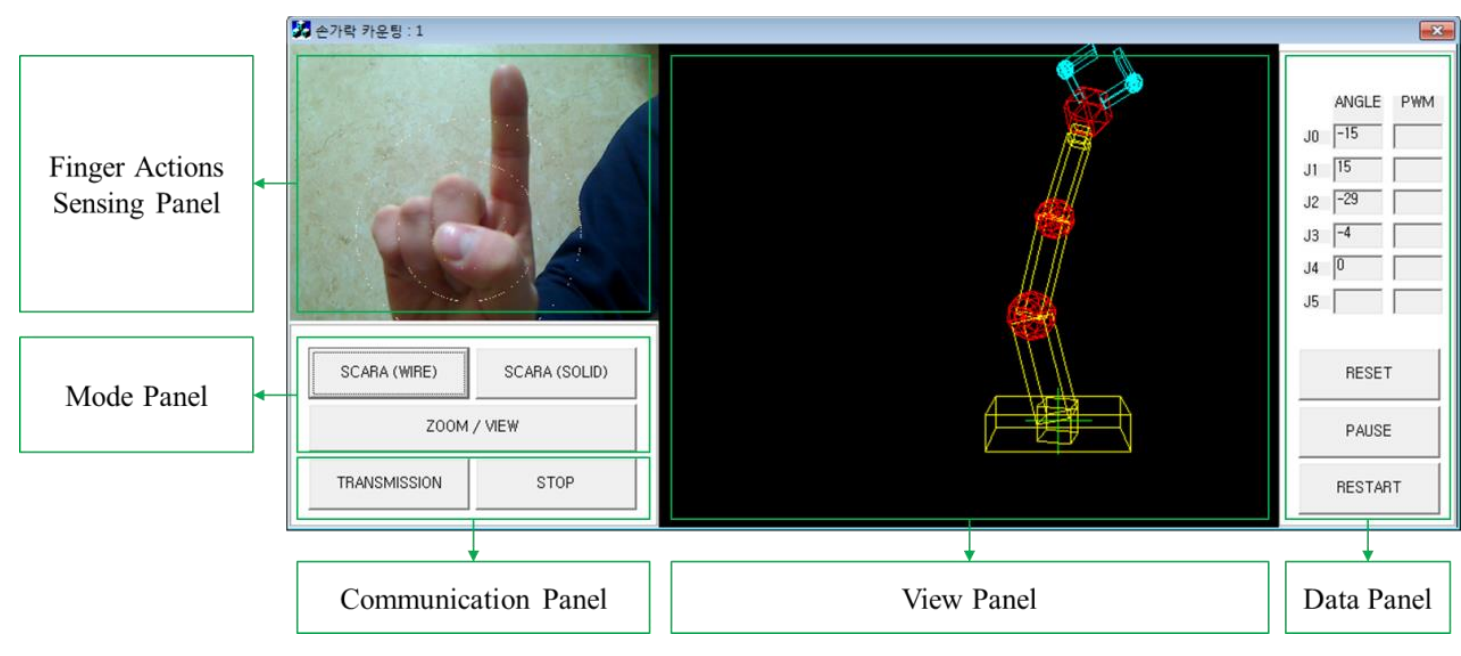

Figure 3. Robot Motion Authoring Tool

The finger actions sensing panel means the finger recognition unit that recognizes the finger actions from the finger)imagestaken by camera. The finger actions recognized by the finger actions sensing panel allows the user to control the industrial robots (such as SCARA, articulated robot, and etc.) displayed on the view panel. The mode panel provides three modes - WIRE, SOLID and ZOOM/VIEW. The WIRE mode allows viewing of robot's internal structure and the SOLID mode only provides robot's external view. In the ZOORIVIEW mode, the view can be zoomed in/out and rotated, allowing the user to view robot's entire structure in detail. The view panel provides the user with a real time 3D-viewing of the robot motion being authored with finger actions sensing. The authored motions can be transmitted to control the robot by clicking TRANSMISSION button, and the actual robot motion can be stopped using STOP button. The data panel provides the virtual robot motion degrees and PWMs to the actual industrial robot in real time. The data panel provides RESET, PAUSE, RESTART button. If the RESET button is clicked, the robot is initialized to the default value If the RESTART button is clicked, the robot is restarted.

\section{Experimental Results and Discussions}

\subsection{Virtual Robot Motion Authoring Simulation using Finger Actions Sensing}

In this section, we present the simulation on a virtual articulated robot motion authoring using the proposed finger actions sensing based robot motion authoring system. The finger actions sensing based robot motion authoring system proposed in this paper was implemented on a personal computer with 1GByte memory, 1.80GHz AMD AthlonTM 64-bit processor CPU and ATI Radeon X1600 graphic card that supports DirectX. As well, Logitech Webcam Pro 9000 was used. The robot motion authoring tool in this paper was implemented with 
OPENGL, the open source programming library. As well, the motion ranges and directions of the virtual articulated robot are almost similar to ones of the actual robot. Figure 4 depicts how a robot part is changed in the WIRE/SOLID mode.

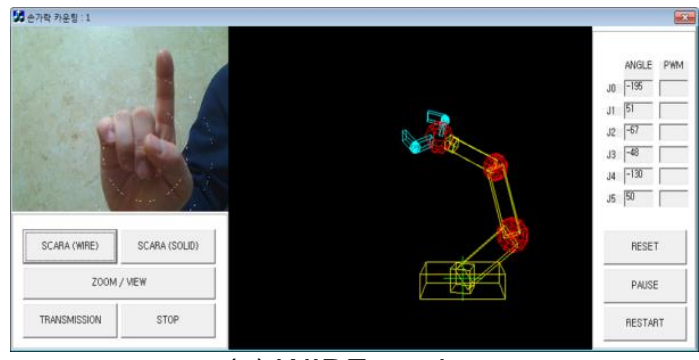

(a) WIRE mode

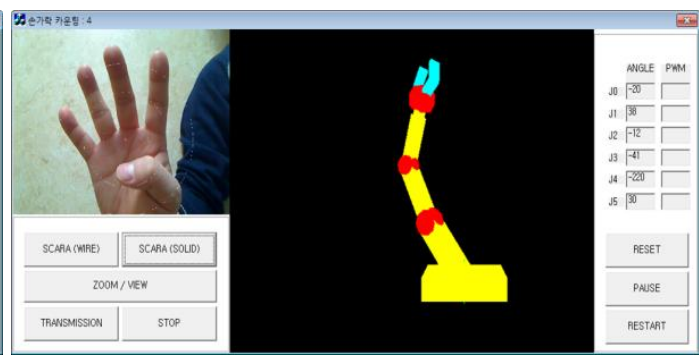

(b) SOLID mode

\section{Figure 4. Robot Motion Authoring Tool}

Table 1 lists the definitions of finger-value event for WIRE, SOLID mode. In WIRE and SOLID mode, internal and external structures of the robot can be yiewed, respectively. In either mode, if the number of fingers recognized by the finger interface is 0 or 4, 1-Axis or 5Axis rotates around the $\mathrm{Y}$ axis. For 1, 2, 3 fingers, 2-Axis, 6-Axis, 4-Axis rotates around the $\mathrm{Z}$ axis, respectively.

\section{Table 1. Definitions of Finger-Value Evvent for WIRE and SOLID Mode}

1-Axis rotation (base $\mathrm{Y}$ )
2-Axis rotation (base Z)
3-Axis rotation (base Z)
4-Axis rotation (base Z)
B. WOLED
5-Axis rotation (base Y)
6-Axis rotation
(Gripper ON/OFF)

Figure 5 depicts how a robot part is downsized in the ZOOM/VIEW mode when the number of finger is recognized as 1 and 2. The ZOOM/VIEW mode allows the user to zoom in and out of the robot structure and vary the camera angle for a detailed view. Similar to the WIRE mode, the camera is rotated clockwise and counter-clockwise for 3 and 4 fingers, respectively, according to the definitions of Table 2 so that the user can view the robot from a desired angle. Accordingly, the user is able to not only create but control motions for a part of a robot based on the number of fingers recognized. 


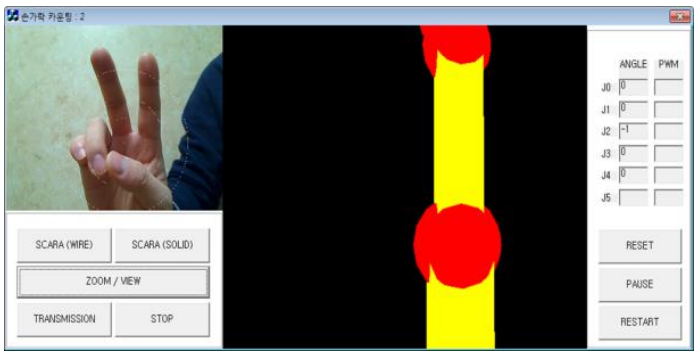

(a) ZOOM IN

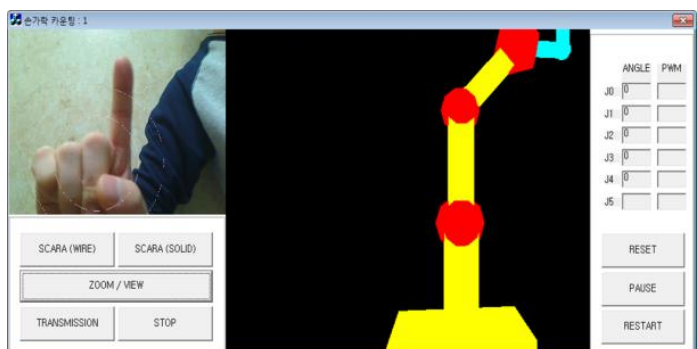

(b) ZOOM OUT

Figure 5. Downsized Robot in ZOOM/VIEW Mode with 1 and 2 Recognized

Also, a robot rotation part is enlarged in the ZOOM/VIEW mode when the number of finger is recognized as 2 and 3. Table 2 list the definitions of finger-valye event for ZOOM/VIEW mode.

Table 2. Definitions of Finger-Value Event for ZOOM/VJEW Mode

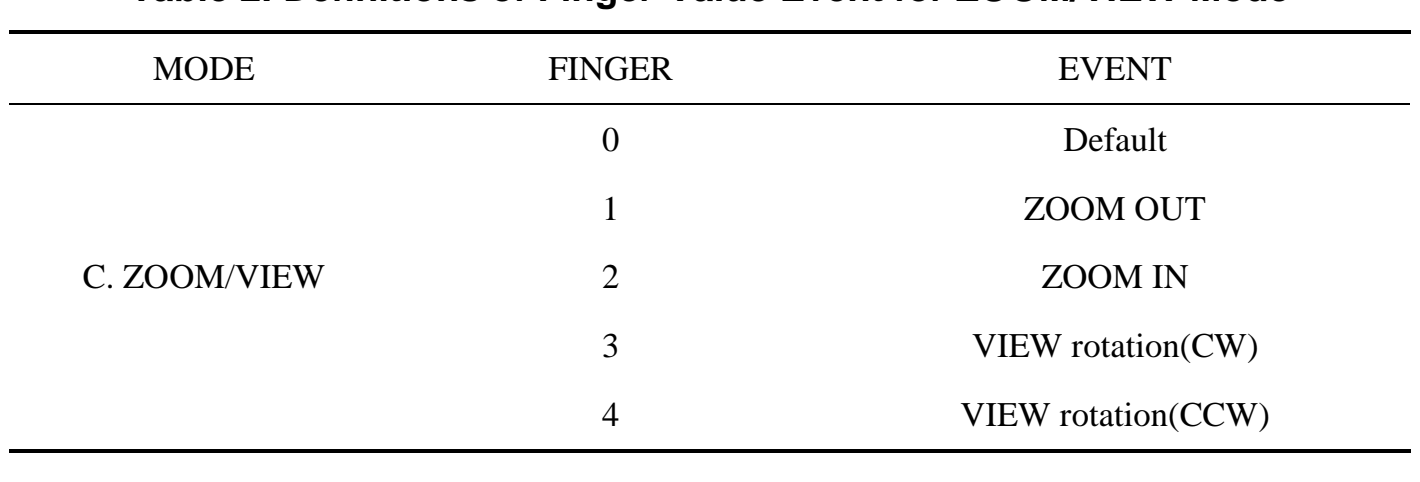

Figure 6 shows the measured virtual robot motion authoring experimental results. The experimental results confirmed that the finger actions sensing based robot motion authoring system proposed by this study provides general users with an intuitive, fun and easy way to create motions.

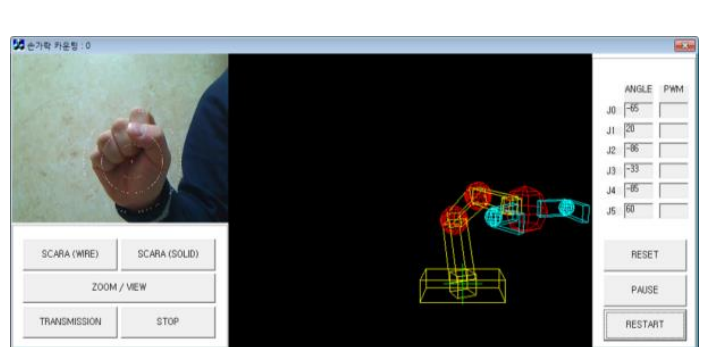

(a) 0 finger

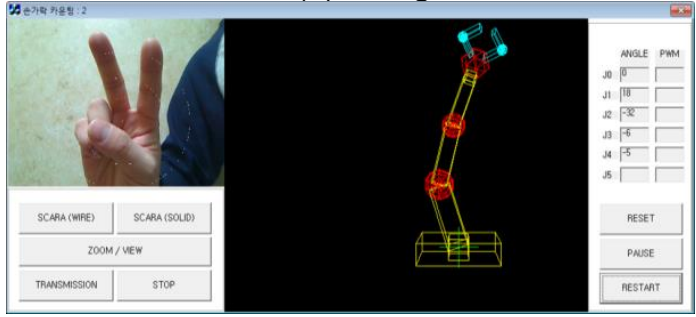

(c) 2 fingers

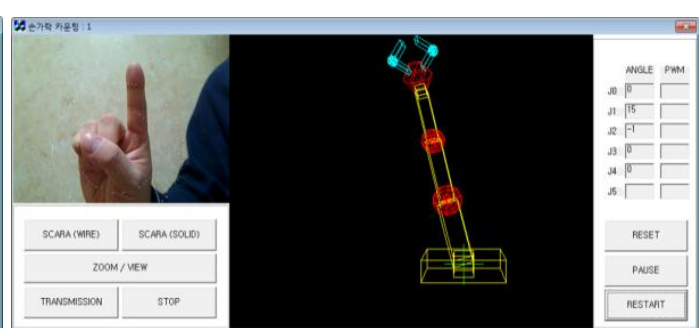

(b) 1 finger

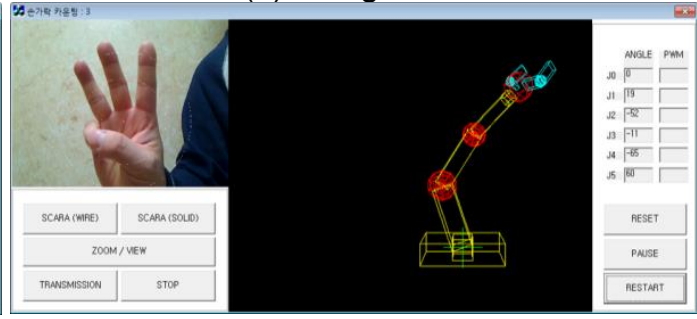

(d) 3 fingers 


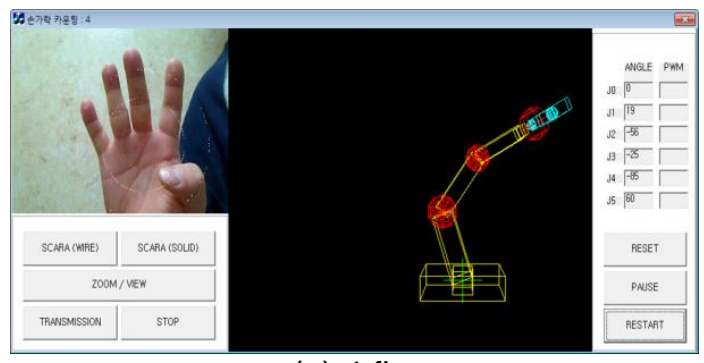

(e) 4 fingers

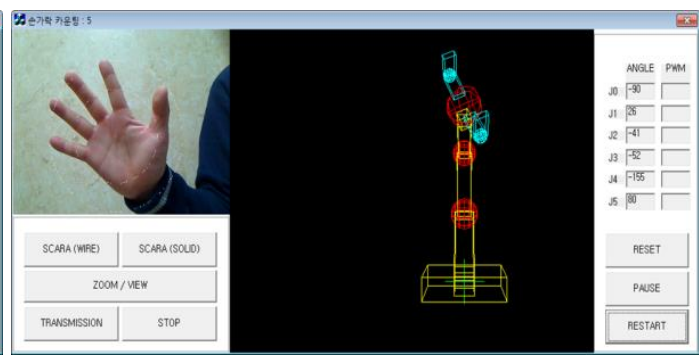

(f) 5 fingers

Figure 6. The Virtual Robot Motion Authoring Experimental Results

\subsection{Actual Articulated Robot Motion Authoring Simulation using Finger Actions Sensing}

The purpose of experiment in 3.2 was to confirm whether or not robot notions authored by the proposed system works with an actual robot well. Acural articulated robot is an miniaturetype robot. The maximum height of the used robot is $536 \mathrm{~mm}$, and the maximum width is $116.5 \mathrm{~mm}$. Also, the maximum arm length is $470 \mathrm{~mm}[13]$. $7 \mathrm{RC}$-seryo motors were used. For control, the robot arm can be easily controlled by a computer or embedded board by using NT-SERVO-16CH-1 and NT-USB2UART. The proposed authoring system as shown in Figure 7 consisted of a robot motion authoring tool, a-PC camera, and an actual articulated robot. The motions authored by the tool were applied to an actual robot for the experiment. The motion data was transmitted to the robot using serial communication and it was examined how the contents produced by the robot motion authoring tool controlled the robot according to user's intention. Figure 7 shows the experimental environment of an actual articulated robot motion authoring simulation.

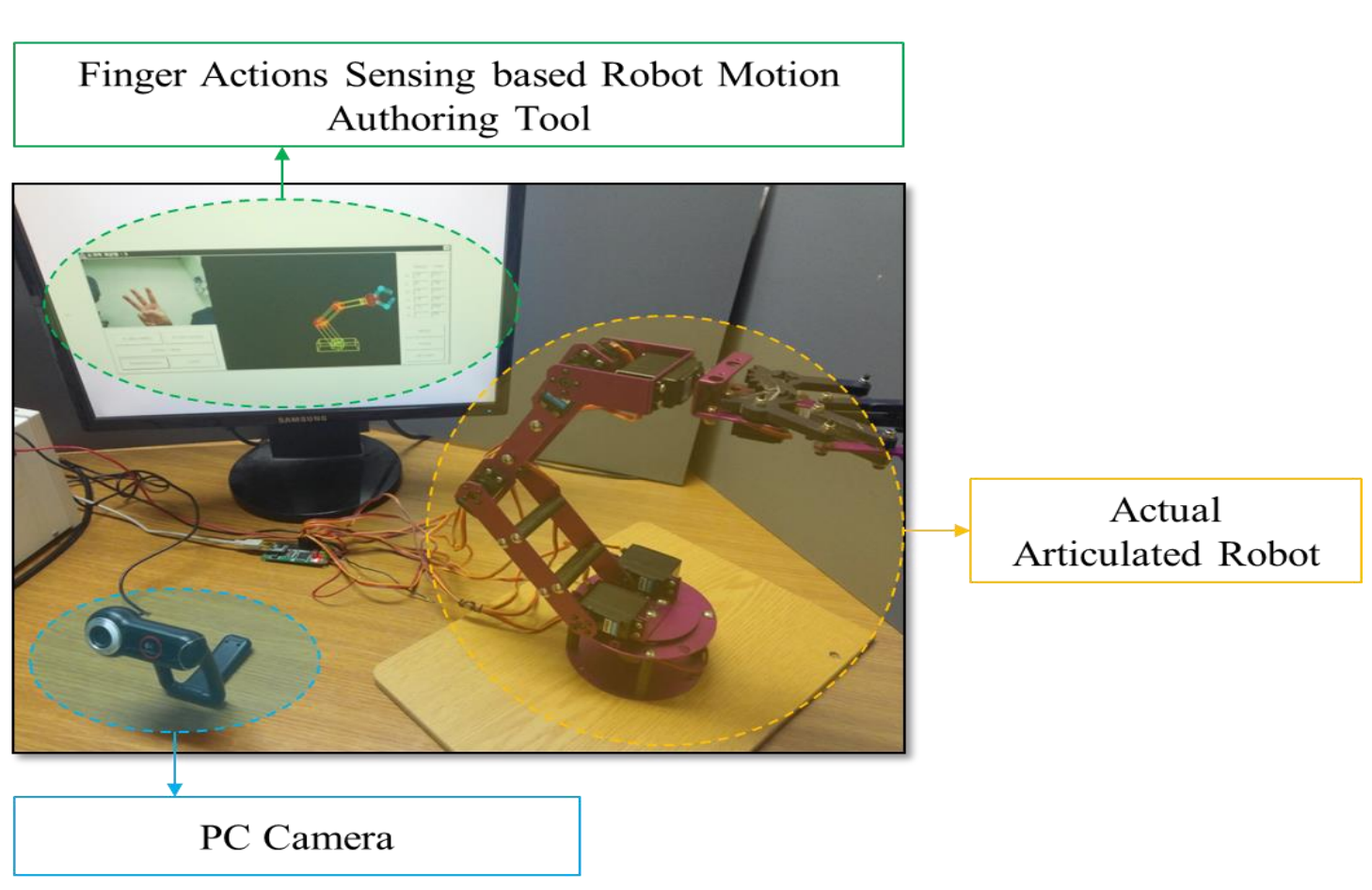

Figure 7. Experimental Environment of an Actual Articulated Robot Motion Authoring Simulation 
The following figures show actual articulated robot motion authoring simulation results on various motions. Also, the following tables show the measured robot motion data for the transmission. In this section, simulation was conducted to measure the robot motion data based on various motions (such as motion \#1, motion \#2, motion \#3, and motion \#4). Figure 8 shows the experimental result of an actual articulated robot motion \#1.

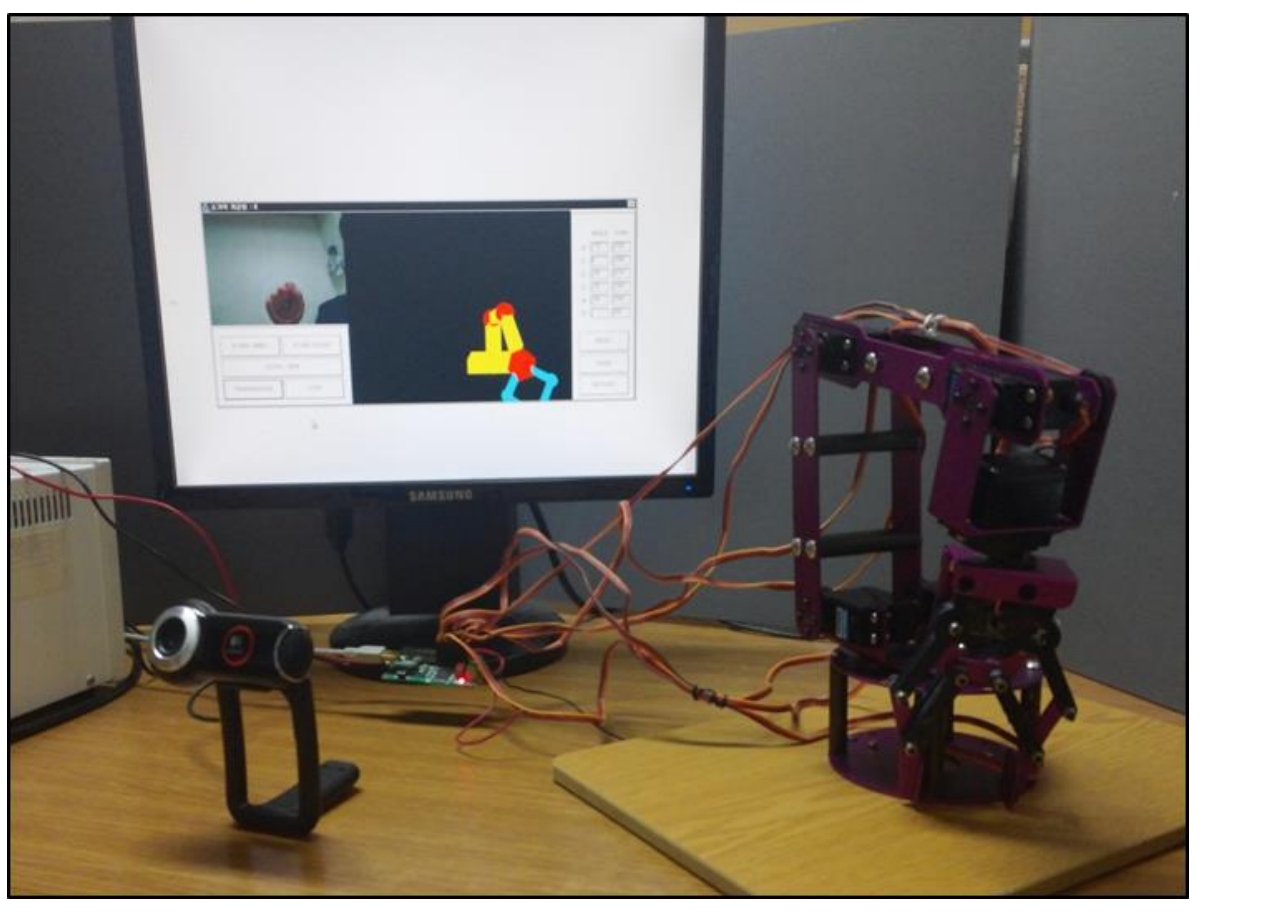

Figure 8. Experimental Áctual Articulated Robot Motion \#1 Result

Table 3 shows the degree of the joint value, and the corresponding PWM value to drive the torque of the brake proportionally to the torque sensed by the proposed finger actions sensing based robot motion authoring system in experimental motion \#1. From the experimental result, we confirmed both the degree and PWM values for motion \#1 authoring using the proposed authoringsystem.

\begin{tabular}{ccc} 
Table 3 . The Result Data of Actual Articulated Robot Motion \#1 \\
\hline CHANNEL & DEGREE & PWM(0700-2300) \\
Ch0 & -70 & 1970 \\
$\mathrm{Ch} 1$ & 0 & 1600 \\
$\mathrm{Ch} 2$ & -80 & 2216 \\
$\mathrm{Ch} 3$ & -55 & 1326 \\
$\mathrm{Ch} 4$ & -60 & 1864 \\
$\mathrm{Ch} 5$ & 0 & 700 \\
\hline
\end{tabular}


Figure 9 shows the experimental result of an actual articulated robot motion \#2.

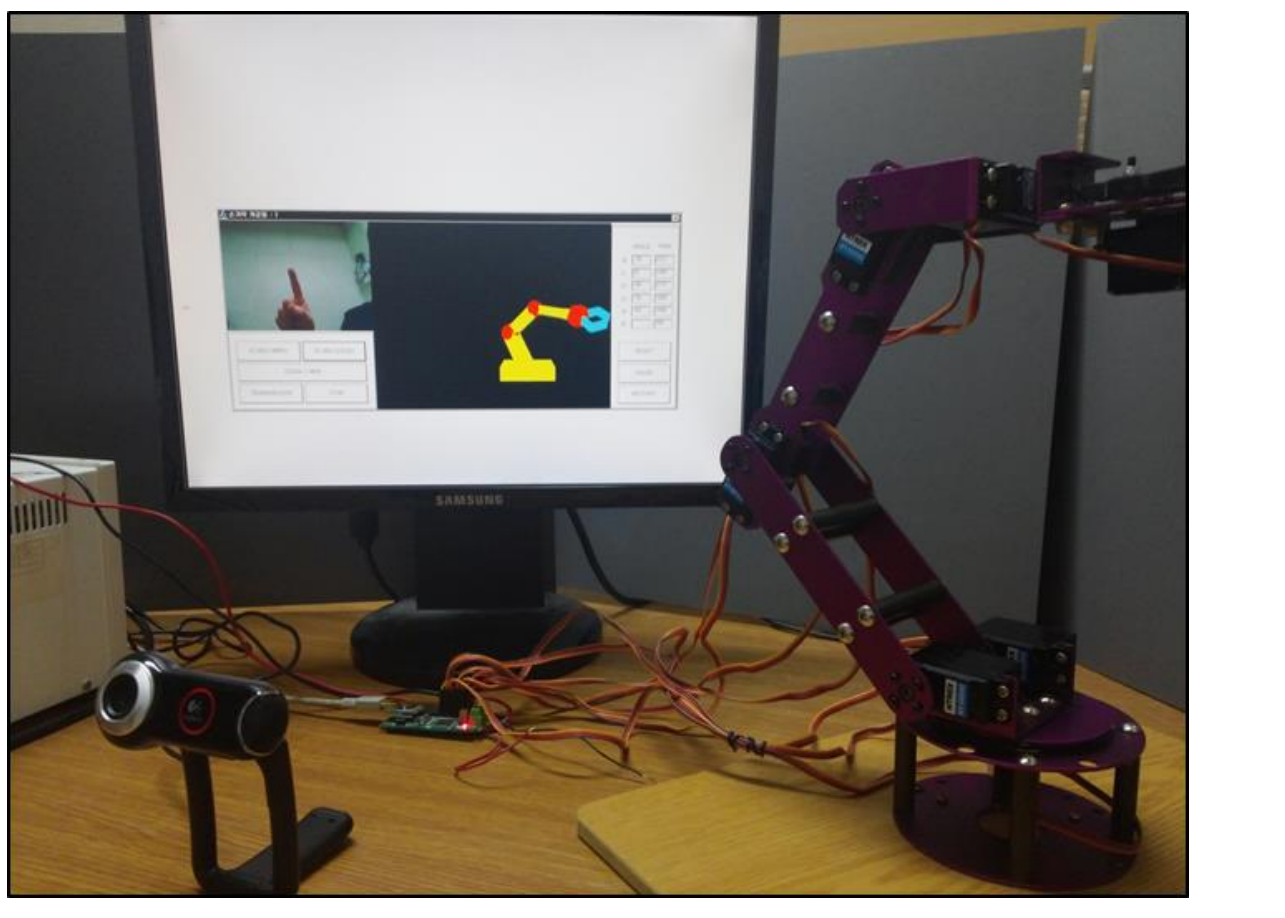

Figure 9. Experimental Actual Articulated Robot Motion \#2 Result

Table 4 shows the degree of the joint value, and the corresponding PWM value to drive the torque of the brake proportionally to the torque sensed by the proposed finger actions sensing based robot motion authoring system in experimental motion \#2. From the experimental result, we confirmed both the degree and PWM values for motion \#2 authoring using the proposed authoring system.

Table 4. The Result Data of Actual Articulated Robot Motion \#2

\begin{tabular}{rlc}
\hline & DEGREE & $\operatorname{PWM}(0700-2300)$ \\
\hline Ch0 & -30 & 2212 \\
$\mathrm{Ch} 1$ & 30 & 1948 \\
$\mathrm{Ch} 2$ & -80 & 2177 \\
$\mathrm{Ch} 3$ & -50 & 1680 \\
$\mathrm{Ch} 4$ & -60 & 1644 \\
$\mathrm{Ch} 5$ & 0 & 700 \\
\hline
\end{tabular}

Figure 10 shows the experimental result of an actual articulated robot motion \#3. 


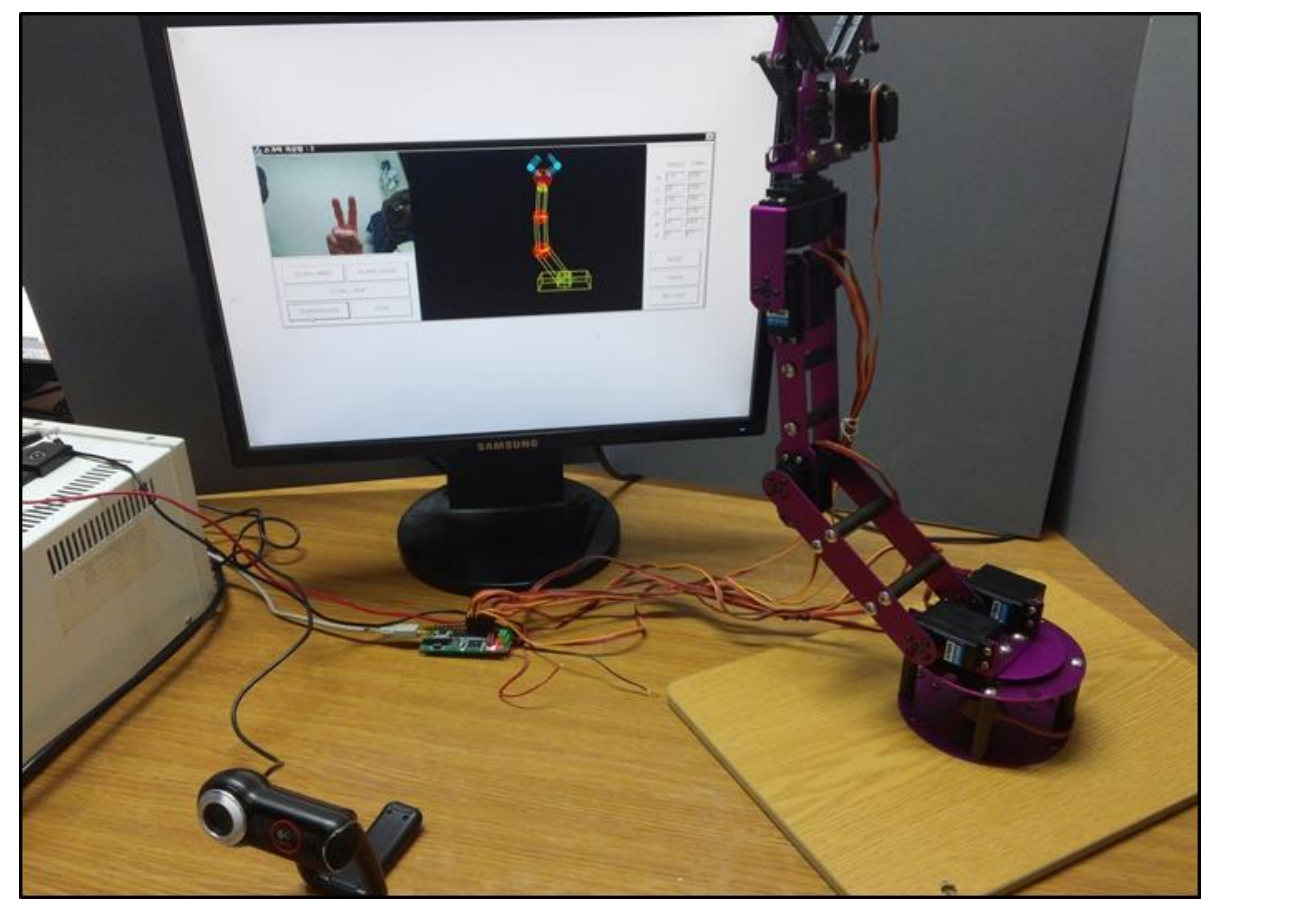

Figure 10. Experimental Actual Árticulated Robot Motion \#3 Result

Table 5 shows the degree of the joint value, and the corresponding PWM value to drive the torque of the brake proportionally to the torque sensed by the proposed finger actions sensing based robot motion authoriag system in experimental motion \#3. From the experimental result, we confirmed both the degree and PWM values for motion \#3 authoring using the proposed authoring system.'

\begin{tabular}{ccc} 
Tab. The Result Data of Actual Articulated Robot Motion \#3 \\
\hline
\end{tabular}

Figure 11 shows the experimental result of an actual articulated robot motion \#4. 


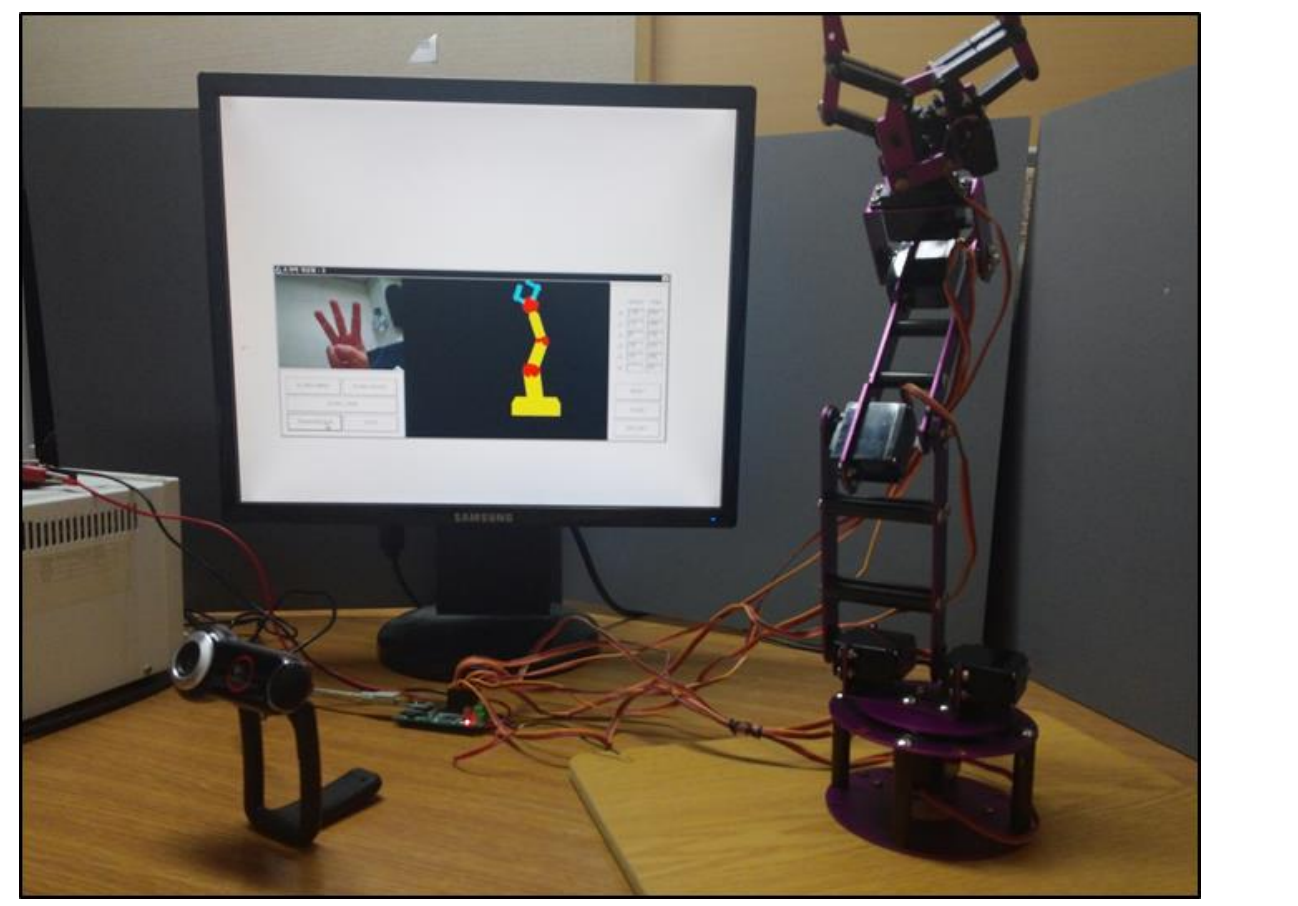

Figure 11. Experimental Actual Artigulated Robot Motion \#4 Result

Table 6 shows the degree of the joint value, and the corresponding PWM value to drive the torque of the brake proportionally to the torque sensed by the proposed finger actions sensing based robot motion authoriag system in experimental motion \#4. From the experimental result, we confirmed both the degree and PWM values for motion \#4 authoring using the proposed authoring system.'

\begin{tabular}{ccc} 
Tabe 6. The Result Data of Actual Articulated Robot Motion \#4 \\
\hline
\end{tabular}

\section{Conclusion}

This paper proposed an authoring system capable of creating and controlling motions of industrial robots based on finger actions sensing. The proposed system is user-friendly and intuitive and facilitates motion authoring of industrial robots using fingers, which is second 
only to language in terms of means of communication. The proposed authoring system also uses graphic motion data to simulate user-created robot contents in a 3D virtual environment so that the user can view the authoring process in real time and transmit the authored robot contents to control the robot. The validity of the proposed robot motion authoring system was examined based on simulations using the authored motion robot contents as well as experiments of actual robot motions. The proposed robot motion authoring method is expected to provide user-friendly and intuitive solutions for not only various industrial robots, but also other types of robots including humanoids.

\section{Acknowledgments}

This paper was supported by the 2013 Education and Research Promotion Program of KoreaTech.

\section{References}

[1] S. Y. Nam, H. Y. Lee, S. J. Kim, Y. C. Kang and K. E. Kim, "A study on design and implementation of the intelligent robot simulator which is connected to an URC system", Institute of Electronics Engineers of Korea, vol. 44, no. 4, (2007), pp. 157-164.

[2] K. H. Seok and Y. S. Kim, "A new robot motion authoring method using HTM", Proceedings of the International Conference on Control, Automation and Systems, (2008), pp. 2058-2061.

[3] K. W. Sok, M. Kim and J. H. Lee, "Simulating Biped Behaviors from Human Motion Data", ACM Transactions on Graphics (SIGGRAPH), vol26, no. 3, Articlel07 (2007).

[4] K. H. Lee, M. G. Choi and J. H. Lee, "Motion Patches:Building Blocks for Virtual Environments Annotated with Motion Data", ACM Transaction on Graphics (SIGGRAPH), vol. 25, no. 3, (2006), pp. 898-906.

[5] L. Reng, T. B. Moeslund and E. Granum, "Finding Motion Primitives in Human Body Gestures", Lecture Notes in Computer Science, no. 3881, (2006), pp. 133, 144.

[6] H. Lee, Y. Tateyama and T. Ogi, "Motion Templates based User Interface for Immersive Virtual Reality Environment", International Joumal of Control and Automation, vol. 5, no. 4, (2012) December, pp. 113-116.

[7] Y. Chen, Y. Zhou1, X, Cheng and Y. Mi, UUpper Limb Motion Recognition Based on Two-Step SVM Classification Method of Surface EMQ̣", International Journal of Control and Automation, vol. 6, no. 3, (2013) June, pp. 249-266.

[8] S. H. Kim, S. M. Lee, S. J. Kim and J. S. Lee, "Object Tracking of Mobile Robot using Moving Color and Shape Information for the agedyalking”, International Journal of Advanced Science and Technology, vol. 3, (2009) February, pp. 59-68.

[9] K. Qian, J. Niu and H. Yang "Developing a Gesture Based Remote Human-Robot Interaction System Using Kinect", J. Smart Home(yøl. 7, no. 4, (2013), pp. 203-208.

[10] F. G. Pereira, R. F. Vassallo and E. O. T. Salles, Human-Robot Interaction and Cooperation through People Detection and Gesture Recognition. J. Control, Automation and Electrical Systems, vol. 24, issue 3, (2013), pp. 187-198.1

[11] A. Licsáfand T. Szirányi, "User-adaptive hand gesture recognition system with interactive training", Image and Vision Computing 23, (2005), pp. 1102-1114.

[12] B. YG. S. Y. Park, Y. S. Kim, I. G. Jeong, S. Y. Ok and E. J. Lee, "Hand Tracking and Hand Gesture Recognition for Human Computer Interaction”, J. Korea Multimedia Society, vol. 14, no. 2, (2011), pp. 182193.

[13] http://www.ntrex.co.kr/ 


\section{Authors}

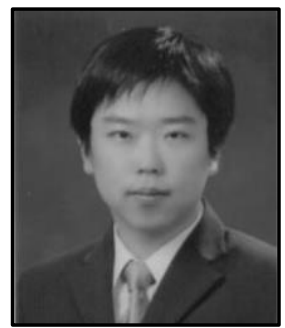

Kwnag-Ho Seok, he received the B.S. degree in internet-media engineering from Korea University of Technology and Education (KOREATECH), Korea, in 2007 and the M.S. degree in Information media engineering, from Korea University of Technology and Education (KOREATECH), Korea, in 2009. Since 2009 to now, he has been Ph.D. student in Humane Interaction Lab (HILab), Korea University of Technology and Education (KOREATECH), Cheonan, Korea. His current research interests include human-robot interaction, and power system.

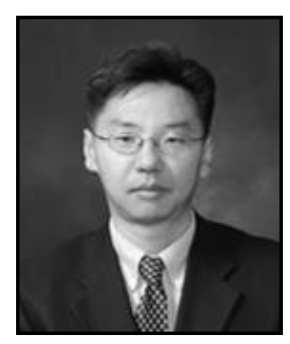

Yoon Sang Kim, he received the B,S. M.S., and Ph.D. degrees in Electrical Engineering from Sungkyunkwan University, Seoul, Korea, in 1993, 1995, and 1999, respectively. From 1999 to 2000 he was a member of the Postdoctoral Research Staff of Korea Institute of Science and Technology (KIST), Seoul, Korea. From 2000 to 2003 he was a Faculty Research Associate in the Department of Eleetrical Engineering, University of Washington,Seattle. Fon 2003 to 2005 he was a Member of the Senior Research Staff Samsung Advanced Institute of Technology (SAIT) Suwon, Korea. Since 2005, he has been an Assqeiate Professoryat the School of Computer Science and - Engineering, KKorea University of Technology Education (KOREATECH), Cheonan, Korea. His current research interests include Virtual simulation, Power-IT technology, and Bioinformatics. Dr. Kim was awarded the Korea Science and Engmeering Foundation (KOSEF) Overseas Postdoctoral Fellow in 2000. He is a member of IEEE, IEICE, ICASE, KIPS, and KJEE.

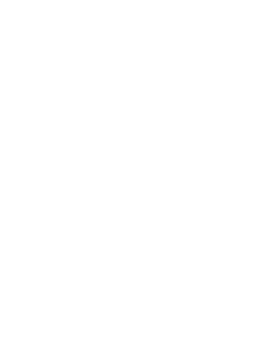

